\title{
Broadening research consent in the era of genome-informed medicine
}

\author{
Courtney Kronenthal, PhD ${ }^{1}$, Susan K. Delaney, MPA ${ }^{1}$, Michael F. Christman, PhD ${ }^{1}$
}

\begin{abstract}
Genetic variant associations and advances in research technologies are generating an unprecedented volume of genomic data. Wholegenome sequencing will introduce even greater depth to current data sets and will propel medical research and development. Yet as one area of biomedical research evolves, another stagnates: informed consent. As presently employed, informed consent is not entirely attuned to the era of whole-genome sequencing. The greatest value of genomic data lays in its accessibility over time; the current model of informed consent restricts the use of data and does not readily accommodate prospective basic and clinical research, a priori research, or opportunities to act upon incidental findings. It also disengages the research participant from the discovery process, discouraging the provision of research results that may have clinical value to that individual. A
\end{abstract}

revisited informed consent approach-the Informed Cohort Oversight Board (ICOB) - has been proven successful at consenting individuals to a model which facilitates the simultaneous construction of longitudinal data with the return of results to participants as scientific knowledge and technology allows. The opportunity to sequence once and consult often is cost-effective, encourages scientific innovation, and provides the opportunity to quickly translate genomics into better clinical care.

Genet Med 2012:14(4):432-436

Key Words: biobanking, genome-informed medicine, incidental findings, informed consent, longitudinal data, pharmacogenomics, personalized medicine, whole-genome sequencing
Advances in understanding genomic variation and associated clinical phenotypes have resulted in more than 1,300 confirmed associations. ${ }^{1}$ When these biomarkers are coupled with clinical information-family history, lifestyle, and other environmental factors-more precise predictions about a person's susceptibility to developing disease and disease progression can be made for conditions including cancers, cardiovascular disorders, and obesity, and for patients' response to therapeutics and prescription medications, including statin therapy and antidepressants. ${ }^{2}$ As scientific understandings advance, so do the technologies that support them. Array-based high-throughput genotyping platforms are giving way to next-generation sequencing, technologies simultaneously increasing in speed and accuracy and decreasing in cost. ${ }^{3,4}$ The translation of genomic information assayed in a laboratory into useful clinical decisions and better health outcomes will become routine, moving genomeinformed, personalized medicine from promise to practice. ${ }^{5,6}$

Discovering new genetic associations, innovating novel treatments, and delivering genome-informed care begin with understanding the biological basis of disease and drug metabolism pathways and how they impact subsets of the population. ${ }^{7}$ Scientists begin their exploration of the human genome with high-quality and highly characterized biospecimens, preferably with appropriate clinical annotation, that are often obtained through biobanks. ${ }^{8}$ For instance, the biobank at Coriell Institute for Medical Research manages millions of cell lines and DNA biospecimens, in addition to plasmids, plasma samples, sera, etc., many with associated phenotypic and clinical data., ${ }^{9,10}$ These collections, several supported by divisions of the National
Institutes of Health, have reached more than 60 countries, have been cited in more than 7,000 peer-reviewed publications, and have supported the HapMap and 1,000 Genomes programs. The availability of biospecimens for research have resulted in many important discoveries, including biomarkers that in turn have provided a number of improved clinical outcomes; however, treatments for diseases like Alzheimer disease and muscular dystrophy remain elusive.

Biospecimens are typically recruited into biobank collections for specific purposes, for example, Parkinson disease research, and the use of these biospecimens and their associated clinical data are defined and agreed upon with the donor at the time of collection. The economic aspect of biobanking must also be noted: the resources required to recruit, annotate, and manage high-quality biospecimens critical to research is considerable. ${ }^{11}$ To maximize this investment, we need to ensure long-term utility of collected biospecimens. By narrowing our agreement with the donor, we have created the unintended consequence of eliminating opportunities for valuable biospecimens to be used in further biomarker research. Similarly, clinical research is overseen by a myriad of regulations, including the Health Insurance Portability and Accountability Act, ${ }^{12,13}$ which prevents research investigators from performing retrospective, chartbased research or prospectively evaluating patients by contacting them for follow-up. However, as science and technology outpace our traditional research methods, it will be essential to reexamine and reinterpret previously collected samples to more fully understand human disease and correlations to clinical interventions. 
It is time to consider an informed consent model that respects the privacy concerns of donors while asking them to make a contribution to research that is more durable, broader in vision, and efficient in mindset. Encouraging research participants to do so will ultimately lessen the cost of basic research and speed innovation.

\section{ADAPTING CONSENT FOR PROGRESSIVE RESEARCH}

We are not the first to challenge the classic model of informed consent by exploring alternative models that facilitate recontacting research subjects. ${ }^{14-17}$ McGuire and McGuire, ${ }^{14}$ seeking to maximize data generated in genome-wide association studies, proposed allowing investigators to form the basis of a case group by first identifying the usually small number of individuals in any given genome-wide association study who bear a particular variation and then assembling a large cohort of these individuals from multiple genome-wide association studies. Kohane et al. ${ }^{18}$ outlined a model to facilitate interaction with research participants over time in what they describe as a collaborative clinical research regime, the "Informed Cohort" (IC). ${ }^{18}$ The IC is enrolled through an extensive informed consent process; individuals then provide a biospecimen for highthroughput measurements and subsequently use a Web-based personally controlled health record for ongoing participation. Interaction and communication with this IC group is governed by an independent, multidisciplinary team called an IC Oversight Board (ICOB), which determines what clinical information is appropriate for the cohort and the best approach for communicating that information. When a genetic signature is successfully associated with a presymptomatic phenotype, the ICOB alerts the entire participant cohort that an approved clinical implication is available. Participants independently decide whether or not to "tune-in" to the newly available information and whether or not to share the results with their health-care provider. The Kohane model aims to respect regulations and privacy concerns while providing the opportunity to make ongoing health-related disclosures beneficial to the individual in the course of the study. The Kohane model also allows participants to share health information in a de-identified format for downstream research such as association studies.

\section{THE ICOB MODEL IN PRACTICE: CORIELL PERSONALIZED MEDICINE COLLABORATIVE}

Kohane's innovative IC model was put into practice in 2007 through the Coriell Personalized Medicine Collaborative (CPMC), a prospective, observational research study seeking to determine the utility of personal genome information in health management and clinical decision-making while also educating patients, medical professionals, and the public on genomics and personalized medicine (http://www.cpmc.coriell.org). The study also aims to contribute to the evidence-based research required to inform policies and regulations, e.g., third-party payer practices; encourage the development of companion diagnostics; integrate genomic data into both electronic medical records and clinical decision-support tools; and establish recommendations for best practices. A true collaborative, the CPMC study is a multiparty effort of volunteer study participants, physicians, scientists, ethicists, genetic counselors, pharmacists, information technologists, and hospital and academic partners.

An overview of the CPMC study and an explanation of genetic risk estimates have been described previously. ${ }^{19,20}$ In brief, the CPMC study is currently composed of three cohorts: community, cancer, and heart disease. Individuals are recruited through cohort-specific mechanisms, but all are consented through a comprehensive informed consent process before providing a saliva sample for DNA analysis in Coriell's Genotyping and Microarray Center using Clinical Laboratory Improvement Amendments-certified genotyping platforms (Figure 1). Study participants then activate their personal CPMC Web portal account and complete detailed online questionnaires that capture demographic details (e.g., gender, age, ethnicity), medical history (e.g., hospitalizations, allergies), lifestyle (e.g., doctor visits, physical activity, alcohol use), and family medical history (e.g., congenital disease). Electronic medical record data, such as tumor registry information for cancer cohort participants, are also collected.

A combination of the study participant's self-reported questionnaire data and the lab-analyzed genomic data is presented in the form of personalized risk reports that identify genetic variant results and when possible, nongenetic risk factors (e.g., lifestyle risk) for conditions that are, at minimum, potentially actionable. A potentially actionable condition is defined as a condition for which risk can be mitigated by individual action (behavior and lifestyle) or by medical action (screening, medication, preventive treatment, or early intervention). Results include complex disease and pharmacogenomic (PGx) results, and are made available to study participants on an ongoing basis via the CPMC Web portal. When a new result is available, study participants receive an e-mail notification; the e-mail correspondence includes generalities about the condition but never any personal risk information. Participants can then sign into their personal Web portal accounts and decide whether or not to view each personalized result. If they select the "View My Result" option for, as an example, coronary artery disease, they are first directed to an educational page about coronary artery disease, including an anticipatory guidance video, before accessing their personalized risk results. This information flow is designed to encourage participants to educate themselves on a condition or medication before viewing their personalized results. Educational pages can be accessed even before one decides whether or not to view a personal result, as they are available on the public study site. If participants decide not to view a specific result-by not signing into their CPMC study accounts or by selecting the "Do Not View My Result" option once signed in-they may reconsider and return to the Web portal at a later time to view their personalized results. Supplemental to the online education materials, study participants and their physicians have access to Coriell's board-certified genetic counselors, at no cost. They also have access to PGx-trained pharmacists through collaboration with the 


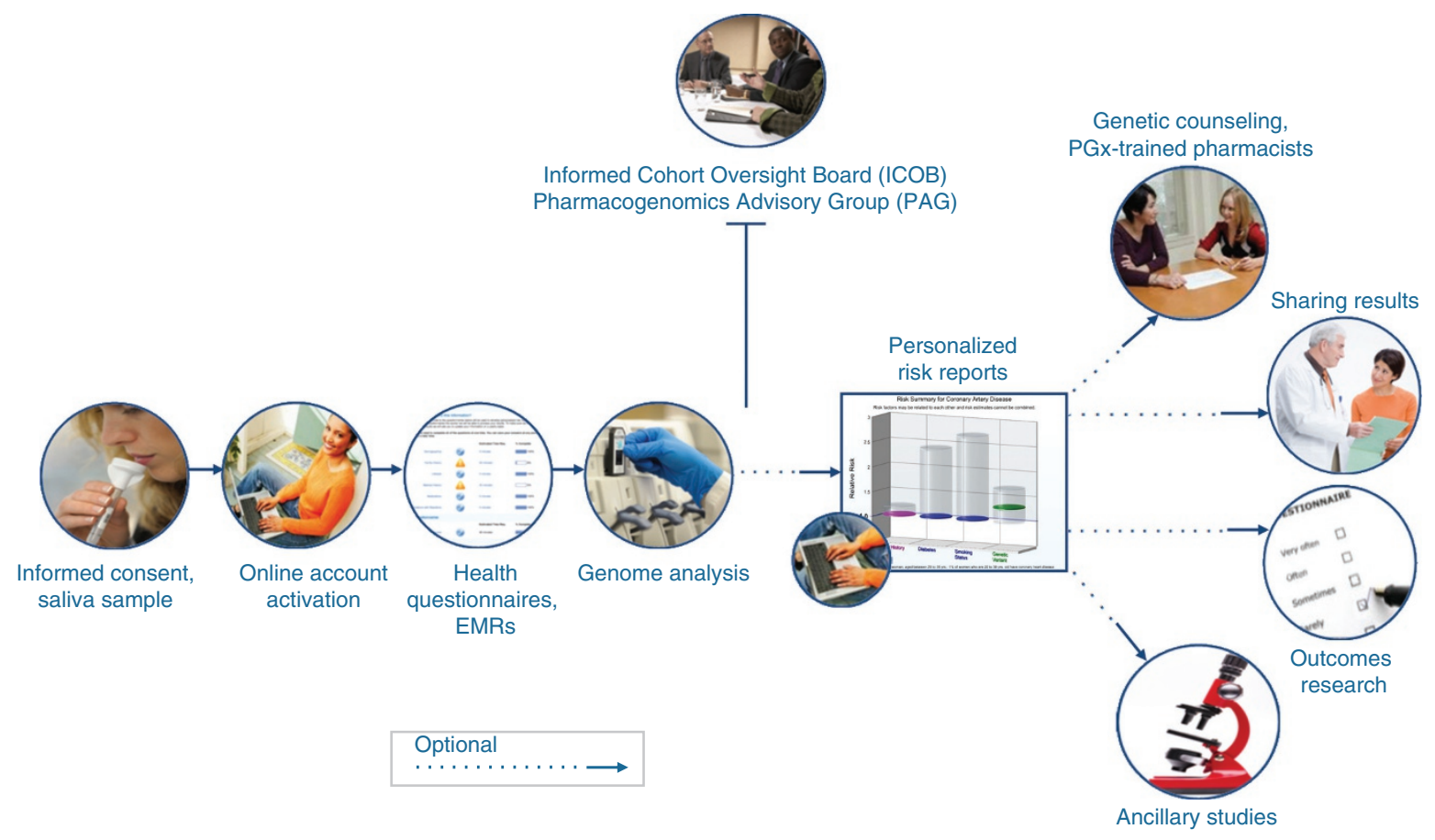

Figure 1 Coriell Personalized Medicine Collaborative (CPMC) Research Study. After providing consent and a saliva sample for genotyping in Coriell's lab (CLIA-certified), and completing detailed health questionnaires, CPMC participants have access to personalized risk reports identifying genetic variant results and when possible, nongenetic risk factors. Study participants decide whether or not to view each potentially actionable, ICOB-approved result and can contact, at no cost, CPMC genetic counselors and pharmacogenomics-trained pharmacists. Participants are encouraged to share their risk report information with their physicians and complete outcome surveys to contribute to the evidence needed to determine the utility of genetic information in clinical care. EMR, electronic medical record; PGx, pharmacogenomic.

American Pharmacists Association. Participants are reminded of these counseling opportunities each time a new result is available and throughout the study in an effort to encourage education both before and after they view their results. The CPMC's genetic counseling and pharmacist resource is a unique aspect of the study, which places keen emphasis on the understanding of genomic data and their clinical relevance. Physicians' and their patients' understanding of how to use clinically relevant genetic data will be critical to the adoption of personalized medicine, and the establishment of best practices in its delivery.

Participants are encouraged to share their personalized risk report information with their physicians for clinical decisions. They are also asked to complete periodic Web-based outcome surveys to provide insight into whether viewing one's personalized risk information, including genomic information, influences personal health decisions, clinical care decisions, and overall health. Study participants can also opt into sharing their de-identified data with the scientific community for research purposes. The structure of the CPMC study allows Coriell to build a cohort with rich genotypic and phenotypic data with which to discover genetic variation that affects drug toxicity and efficacy, as well as to discover currently unknown gene variants that elevate a person's risk of complex diseases. The CPMC study will be making longitudinal data sets available to the research community as the study progresses.

Given the complex nature of multifactorial diseases, the delicate nature of medication dosing, and the early stages of using genomic associations and risk models in clinical decisions, it was understood that results provided to study participants through the CPMC study needed to be statistically robust and validated, and potentially actionable. Crucial to the CPMC study design was the need for an independent oversight board, separate from the institutional review board, with varied expertise to make these determinations. The CPMC research study adopted a Kohane-like governance model called the CPMC ICOB, which is composed of scientists skilled in genetic research, medical professionals familiar with the use of genetics in medical care, an ethicist, and a community leader (http://www.cpmc.coriell.org). The CPMC ICOB evaluates health conditions and genetic variants for inclusion in the CPMC study to determine whether the condition is, at minimum, potentially actionable and to establish the scientific validity of nominated genetic variants as risk factors for the condition.

As the CPMC study prepared to return PGx risk reports, a second advisory group was convened with specialized PGx expertise; the CPMC's Pharmacogenomics Advisory Group is composed of pharmacists, pharmacologists, clinicians with experience in PGx, and an ethicist (http://www.cpmc.coriell.org). The Pharmacogenomics Advisory Group reviews the medications where efficacy or adverse events are known to be affected by genetic variation and they vote on whether drug-gene pairs are potentially actionable. The opinions of the Pharmacogenomics Advisory Group are presented to the ICOB for approval. In cases where the ICOB finds a particular risk factor (genetic or 
nongenetic) to be actionable, risk reports and targeted communications are developed and disseminated by the CPMC study team to inform participants of the availability of new disease or drug response results.

To date, the ICOB has approved 29 health conditions and PGx responses in the form of drug-gene pairs. The board has not approved submissions, including those related to atrial fibrillation, citing non-actionability due to the unfavorable risk-to-benefit ratio of improved health outcomes. They based their decision on the current limited ability to screen for the condition, lack of clear guidelines for risk mitigation, and no reduction in time to diagnosis. If these limitations diminish as time passes, the ICOB will revisit this condition and revote on its potential actionability. Breast cancer was initially voted as non-actionable because the radiation risk imposed by preventative screening outweighed the candidate's genetic variant risk. When new variant information became available and was validated, it was reevaluated by the ICOB and voted as actionable.

\section{DISCUSSION}

Scientists have long gathered and preserved biospecimens from patients for research, and many discoveries have been made because of biospecimen availability and the understanding they have afforded the scientific community. However, many of these biospecimens were collected via an essentially traditional consent: approving their use for singular, specific purposes/ processes; acknowledging no further contact with the donor; and breaking any identifying link back to the donor. Not only does this model limit scientific discovery by restricting the range of the specimen's use, it also ignores the donor as part of the discovery process by preventing researchers from recontacting donors when new discoveries, e.g., incidental findings, are made during the study that may be of interest to, or even affect the lives of, donors. Two assumptions are now clear: (i) the greatest value of a biospecimen will be extracted over time as scientific associations are discovered, technological advances are made, and long-term clinical data are collected, and (ii) the return of research results to subjects-an element relatively new to research studies-is welcomed. ${ }^{21,22}$ The time has come to rethink and adapt the current consent process to release utilization constraints while keeping confidentiality and privacy to the utmost standards.

A model anchored by the expertise of an external governance board has proven successful within the context of the CPMC research study. Study participants agree, via their informed consent document, that the ICOB will decide which genetic variants will be made available to them as part of the study. The consent form explains that the study will not provide results on all genetic variants, only those that the ICOB has determined are potentially actionable or actionable. In this approach, study participants are consenting to have access to personalized risk reports for health conditions and medications not available, nor known, today. As new associations are made and potentially actionable conditions are approved by the ICOB, new personalized risk reports are made available to all participants. The model also allows the study to apply new knowledge, including more robust associations and/or improved clinical benefits, to previously rejected candidate variants/conditions; in this manner an element of flexibility and longevity is imposed on the study. In addition, the structure of the CPMC study allows for the collection of longitudinal data sets inclusive of genotypic/ phenotypic information, medical and family health history, lifestyle behaviors, and other electronic medical record data, which are made available in a de-identified format for use by the biomedical research community. Moreover, the ICOB is transparent in its decision-making, allowing for communication within the scientific community-and if necessary, further discussions by the ICOB regarding voted non-actionability of conditions.

As whole-genome sequencing becomes routine, vast amounts of data will be generated, most of which will be poorly understood at the outset. However, banking genetic and medical data as they are generated will provide opportunities to sequence once and consult often, leading to the development of clinical applications to benefit patients. When presented with pertinent research results, CPMC participants' responses on how their lifestyle behaviors change and whether they choose to share their results, and with whom, are revealing a use for genetic information in clinical care. For example, 3 months after viewing their genetic and nongenetic risk results for coronary artery disease, study participants were queried through an online survey about their perceived risk and decision to share results with their health-care provider. Preliminary findings suggest that the perception of one's risk influences the behavioral decisions, including sharing results with health-care providers. ${ }^{23}$

The CPMC has a unique study design with a focus on education, ethical conduct, and scientific validity; the journal Nature described the study as "leading by example,"24 and MIT Technology Review placed the CPMC on its 2010 "Top 10 Research Projects to Watch" list. ${ }^{25}$ The ICOB model seen in practice through the CPMC research study is becoming an example of trusted best practice to concurrently recruit subjects and build large cohorts from which to study longitudinally. This approach will be critical in the era of whole-genome sequencing when we do not know a priori what information will be returned to study subjects; results will be determined as clinically relevant associations are discovered and validated. Moving into health-care delivery, the juxtaposition of the continuing decline in the costs of whole-genome sequencing and the continuing increase in the knowledge of the human genome should encourage the use of biomarkers for dosing medication and disease diagnoses. It is anticipated that third-party payers and providers will recognize the cost-benefit advantage of adopting whole-genome sequencing and the data it can provide for optimal health outcomes, thus driving patient and physician demand for personalized medicine. ${ }^{26}$

National Institutes of Health Director Francis S. Collins has said, "Somehow we've built a network that is so complex and so restrictive and so difficult to deal with that it's beginning to get very hard to do research. The public has an interest in the 
research getting done as well, and perhaps we need to reconsider whether we've got the balance right here or whether there are actions that could be taken that would make it more feasible to undertake large-scale studies of the sort that we really need." ${ }^{27} \mathrm{By}$ rethinking the traditional research consent and implementing a novel informed consent model that is permitting of regular and meaningful engagement with study participants, research studies will be able to continually add value and maximize research dollars over time and as scientific advances are made.

\section{Ethics}

Appropriate institutional review board approval has been obtained. In addition, informed consent has been obtained from the participants involved in the research study.

\section{ACKNOWLEDGMENTS}

The work of the ICOB has been supported by a grant from the RNR Foundation and the Coriell Institute for Medical Research. Preparation of this article for this special issue, "Managing Incidental Findings and Research Results in Genomic Research Using Biobanks \& Archives," and presentation at the conference of the same title was supported in part by the National Institutes of Health National Human Genome Research Institute grant no. 2-R01-HG003178 (S. Wolf, principal investigator).

\section{DISCLOSURE}

The authors declare no conflict of interest.

\section{REFERENCES}

1. Hindorff LA, Junkins HA, Hall PN, Mehta JP, Manolio TA. A catalog of published genome-wide association studies. http://www.genome.gov/ gwastudies. Accessed 22 September 2011 (SNP-trait associations limited to those with $P$ values $<1.0 \times 10^{-5}$ ).

2. Offit K. Personalized medicine: new genomics, old lessons. Hum Genet 2011;130:3-14.

3. Lerner-Ellis JP, Ellis JD, Green R. Direct-to-consumer testing: What's the prognosis? GeneWatch 2010;23:6-7

4. Wetterstrand KA. DNA sequencing costs: data from the NHGRI large-scale genome sequencing program. http://www. genome.gov/sequencingcosts. Accessed 22 September 2011.

5. Link DC, Schuettpelz LG, Shen D, et al. Identification of a novel TP53 cancer susceptibility mutation through whole-genome sequencing of a patient with therapy-related AML. JAMA 2011;305:1568-1576.
6. Welch JS, Westervelt P, Ding L, et al. Use of whole-genome sequencing to diagnose a cryptic fusion oncogene. JAMA 2011;305:1577-1584.

7. Manolio TA, Collins FS, Cox NJ, et al. Finding the missing heritability of complex diseases. Nature 2009;461:747-753.

8. Hewitt RE. Biobanking: the foundation of personalized medicine. Curr Opin Oncol 2011;23:112-119.

9. Coriell Cell Repositories. http://www.ccr.coriell.org.

10. Vaught J, Rogers J, Myers K, et al. An NCl perspective on creating sustainable biospecimen resources. J Natl Cancer Inst Monographs 2011;2011:1-7.

11. Vaught J, Rogers J, Carolin T, Compton C. Biobankonomics: developing a sustainable business model approach for the formation of a human tissue biobank. J Natl Cancer Inst Monographs 2011;2011:24-31.

12. Regulations for the Protection of Human Subjects (45 CFR 46).

13. Health Insurance Portability and Accountability Act (1996), P.L.104-191.

14. McGuire SE, McGuire AL. Don't throw the baby out with the bathwater: enabling a bottom-up approach in genome-wide association studies. Genome Res 2008;18:1683-1685.

15. Lunshof JE, Chadwick R, Vorhaus DB, Church GM. From privacy to open consent. Nat Rev Genet 2008:9:406-411.

16. Caulfield T, McGuire AL, Cho M, et al. Research ethics recommendations for whole-genome research: consensus statement. PLoS Biol 2008;6:e73.

17. Hudson KL. Genomics, health care, and society. N Engl J Med 2011:365:1033-1041.

18. Kohane IS, Mandl KD, Taylor PL, et al. Medicine. Reestablishing the researcher-patient compact. Science 2007;316:836-837.

19. Keller M, Gordon ES, Stack CB, et al. The Coriell Personalized Medicine Collaborative: a prospective study of the utility of personalized medicine. Personalized Med 2010;7:301-317.

20. Stack CB, Gharani N, Gordon ES, Schmidlen T, Christman MF, Keller MA Genetic risk estimation in the Coriell Personalized Medicine Collaborative. Genet Med 2011;13:131-139.

21. Shalowitz DI, Miller FG. Communicating the results of clinical research to participants: attitudes, practices, and future directions. PLoS Med 2008;5:e91.

22. Christensen KD, Roberts JS, Shalowitz DI, et al. Disclosing individual CDKN2A research results to melanoma survivors: interest, impact, and demands on researchers. Cancer Epidemiol Biomarkers Prev 2011;20:522-529.

23. Gordon ES, Stack CB, Gharani N, Schmidlen TJ, Christman MF, Keller MA. Perceived risk, anxiety and sharing behavior in response to personalized risk information in a cohort study. From the 60th Annual Meeting of the American Society of Human Genetics, Washington, DC, November 2010

24. Prainsack B, Reardon J, Hindmarsh R, Gottweis H, Naue U, Lunshof JE. Personal genomes: misdirected precaution. Nature 2008; 456:34-35

25. MIT Technology Review. Briefing: Personalized Medicine. Research to Watch, March/April 2010. http://www.technologyreview.com/biomedicine/24638/

26. West $D$. Enabling personalized medicine through health information technology. Center for Technology Innovation at the Brookings Institute, 28 January 2011. http://www.brookings.edu/papers/2011/0128_personalized_medicine_ west.aspx

27. Collins FS. Future directions in genetic and genomic research. Presented at the SACGHS Meeting, 11-12 June 2003. 